\title{
MUTATIONAL ANALYSIS OF MYBPC3 GENE IN DILATED CARDIOMYOPATHY PATIENTS IN NORTH INDIAN POPULATION
}

\author{
Rubi Bhola*, Om Shankar**, Rashmi Gupta*, Preeti Kumari*, Royana Singh* \\ *Department of Anatomy, Institute of Medical Sciences, Banaras Hindu University Varanasi, UP. \\ ${ }^{* \star}$ Department of Cardiology, Institute of Medical Sciences, Banaras Hindu University Varanasi, UP.
}

\begin{abstract}
Introduction: Today, molecular cardiology is characterized by the integration of high-technology laboratory studies and clinical medicine. Molecular genetics has redefined the etiology and diagnostic criteria for numerous diseases and has led to the development of new, individualized treatment regimens for several cardiovascular diseases. Amongst all, dilated cardiomyopathy is the commonest cause of heart failure. This study was conducted to identify the possible genetic change in dilated cardiomyopathy in North Indian population.

Material \& Methods: Blood samples of dilated cardiomyopathy patients were collected from Cardiology OPD, Sir Sunderlal Hospital, Banaras Hindu University. DNA was isolated using salting out method. PCR was done to amplify exons 32, 33 and 34 of MYBPC3 gene. The PCR product was sequenced to detect the mutational changes in Exons 32-34 of MYBPC 3 gene.

Results: There were 65 control samples and 65 DCM samples were collected. Total 76 intronic variations were reported. In three (BHU/15/425, BHU/15/450, BHU/16/89) patients, disease causing pathogenic variant c3624_3625insC (rs397516029, HMGD CD0910628) was reported in MYBPC3 gene. Insertion of G at 47354119_47354120 position was reported that lead to a frameshift mutation in three subjects. Several Missence variants were also reported $47354121 \mathrm{G}>\mathrm{C}, 47353899 \mathrm{C}>\mathrm{T}, 47353715 \mathrm{C}>\mathrm{A}, 47353647 \mathrm{~A}>\mathrm{C}, 47353626 \mathrm{G}>\mathrm{T}$ that are present in coding region and may lead to alteration in protein structure and function.

Conclusion: Evidence from previous study reported that MYBPC3 play important role in cardiac contraction and responsible for pathogenesis of dilated cardiomyopathy. Therefore, the identification of frequent genetic transmission of dilated cardiomyopathy provides an important tool for the study of pathogenesis of this disease, which is a frequent cause of admission to the hospital and of heart failure.
\end{abstract}

Keywords: Dilated cardiomyopathy, myosin binding protein C, sequencing, DNA.

\section{INTRODUCTION}

Disorders of the heart leading to heart failure are leading cause of morbidity and mortality. Since the term "cardiomyopathy" was coined 30 years ago to describe a group of myocardial diseases of unknown cause, which was termed as idiopathic cardiomyopathy [1].

Out of all types of cardiomyopathies, dilated cardiomyopathy (DCM) and hypertrophic cardiomyopathy (HCM) are the two major cardiomyopathies
(Fig. 1). Other clinical cardiomyopathies include restrictive cardiomyopathy, and arrhythmogenic right ventricular cardiomyopathy [2].

DCM is defined by the presence of: a) fractional shortening (FS) less than 25\% (>2SD) and/or ejection fraction less than $45 \%$ (> 2SD); and b) left ventricular end diastolic diameter (LVEDD) greater than 117\% $(>2$ SD of the predicted value of $112 \%$ corrected for age and body surface area, BSA) [3] excluding any known cause of myocardial disease. In the context of a familial DCM, these criteria are used to diagnose the

Address for Correspondence:

Prof. Royana Singh, Department of Anatomy, Institute of Medical Sciences, Banaras Hindu University Varanasi, Uttar Pradesh, India. Mob: 9450545650 Email: singhroyana@rediffmail.com 
proband in a family.
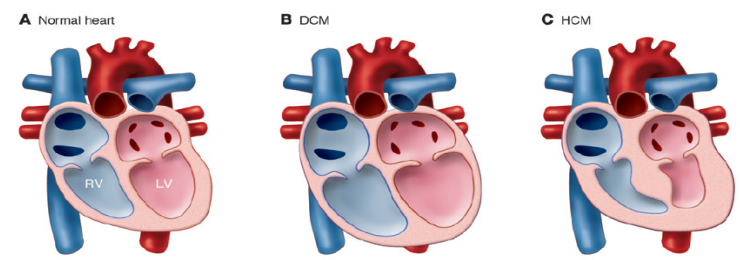

Fig. 1: Morphological changes to the heart in cardiomyopathy. (A) Normal heart. (B) In DCM, the heart enlarges with increased diameter and reduced function. (C) In HCM, the myocardium especially in the LV becomes thickened, leading to impaired filling and emptying [4]

Recent studies report that $20-35 \%$ cases of dilated cardiomyopathy are considered to be familial. Therefore, it is important to study the genetic factors responsible for DCM. Many genes are responsible for DCM out of which the most common are MYBPC3, MYH7, TNNT2, TTN, SGCD. In this study we screened familial DCM patients for the presence of mutation in MYBPC3 gene by DNA isolation and by DNA sequencing [5].

\section{MYBPC 3 (Myosin Binding Protein C) Gene}

The MYBPC3 gene provides instructions for making the cardiac myosin binding protein C (cardiac MYBP$\mathrm{C})$, which contains 35 exons and is found in heart (cardiac) muscle cells. In these cells, cardiac MYBP-C is associated with a structure called the sarcomere, which is the basic unit of muscle contraction. Sarcomeres are made up of thick and thin filaments. The overlapping thick and thin filaments attach to each other and release, which allows the filaments to move relative to one another so that muscles can contract. Regular contractions of cardiac muscle pump blood to the rest of the body [6].

In cardiac muscle sarcomeres, cardiac MYBP-C attaches to thick filaments and keeps them from being broken down. Cardiac MYBP-C has chemical groups called phosphate groups attached to it; when the phosphate groups are removed; cardiac MYBP-C is broken down, followed by the breakdown of the proteins of the thick filament. Cardiac MYBP-C also regulates the rate of muscle contraction, although the mechanism is not fully understood [7].

\section{Chromosomal Location}

Cytogenetic location: $11 \mathrm{p} 11.2$, which is the short $(p)$ arm of chromosome 11 at position 11.2 (Fig. 2).
Molecular location: base pairs $47,331,406$ to $47,352,702$ on chromosome 11 (Homo-sapiens Annotation Release 108, GRCh38.p7) [7].

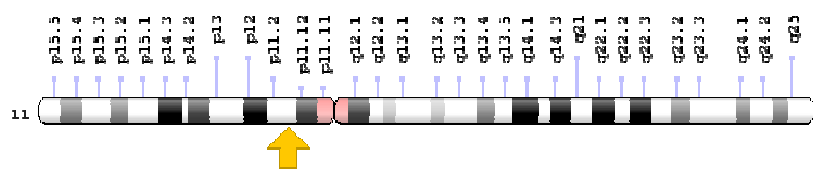

Fig. 2: Cytogenetic location of MYBPC3 gene

The main objective of our study was to evaluate mutational changes in genes MYBPC3 responsible or causing DCM as well as HCM in the population of North Indians from Eastern Uttar Pradesh region.

\section{MATERIAL AND METHODS}

\section{Sample Collection}

3 to $5 \mathrm{ml}$ of peripheral blood was collected in EDTA coated vials after taking inform consent from patients of dilated cardiomyopathy, samples were collected from the Outpatient Department of Cardiology, Sir Sunderlal Hospital of Institute of Medical Sciences, Banaras Hindu University, Varanasi (from 2015-2017). Echocardiogram (ECG) and clinical history of patient were noted; ischemic dilated cardiomyopathy patients were excluded from this study. The study protocol was approved by Ethical Committee of SS hospital, BHU, Varanasi.

\section{DNA Extraction and PCR Amplification}

DNA isolation was done by "Salting out method" and dissolved in TE buffer. Patient samples can be readily checked for concentration and quality using the Nano Drop 1000 Spectrophotometer. After DNA extraction coding region of exons 32-34 were amplified by using following primers exon 32-34F 5'GGCTCAGCCACTGACTTGT 3'and 32-34R 5' AGGGGCCTAGCTTTGTGTG 3' after amplification $5 \mu \mathrm{l}$ PCR amplified products were loaded with DNA loading dye to check the amplification.

\section{DNA Sequencing}

When PCR amplification was complete, PCR products was purified with Exo Sap reagent. The purified PCR products were then sequenced using the sequencing kit and sequencer from Applied Biosystems, USA. Sequencing reactions were analysed using $3130 \mathrm{xL}$ Genetic Analyzer (Applied Biosystem R). Sequencing files were obtained from the 3130xL Genetic Analyzer (applied Biosystems R) were analysed using FinchTV 
viewer. Further analysis was done using MEGA 6 software and Mutation Taster software.

\section{OBSERVATIONS AND RESULTS}

Total of 65 patients samples were collected out of which 50 were male and 15 were female. Agrarose gel electrophoresis results (Fig. 3-5) and sequencing results (Fig. 6,7) exhibited various synonymous and non-synonymous mutations (Table 1). A disease causing pathogenic variation was found in one of the patient.

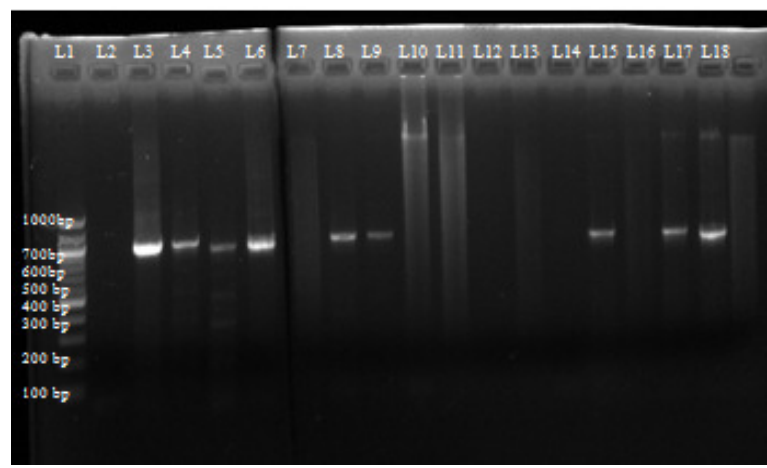
L1: Ladder
L2: NC
L3: BHU/15/407
L4: BHU/15/408
L5: BHU/15/423
L6: BHU/15/424
L7: EMPTY
L8: BHU/15/425
L9: BHU/15/464
L10: BHU/15/465
L11: BHU/15/511
L12: $\mathrm{BHU} / 15 / 512$
L13: BHU/15/513
L14: BHU/16/8
L15: BHU/16/9
L16: BHU/16/58
L17: BHU/16/59

Fig. 3: 2\% Agarose Gel Electrophoresis for MYBPC3 having amplicon size 953 bp

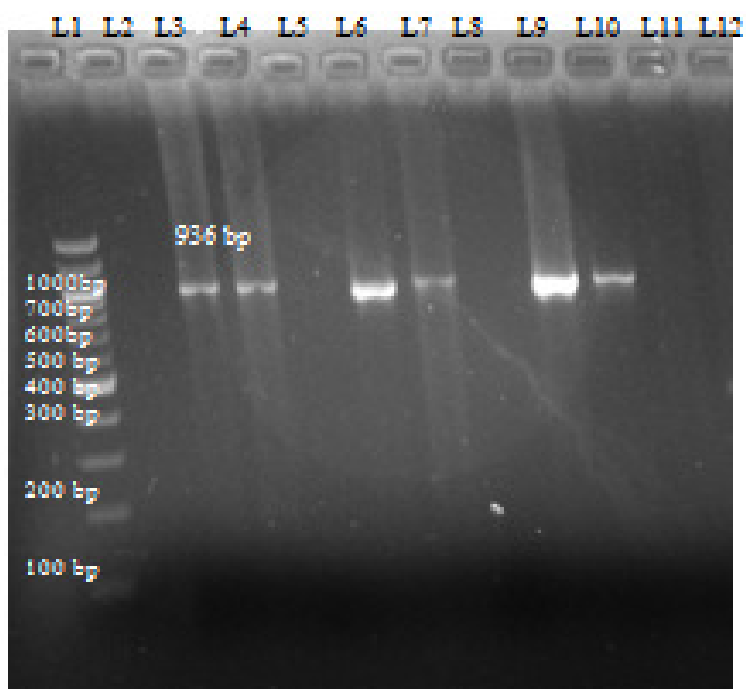
L1: Ladder
L3: BHU/15/464
L2: NC
L5: $\mathrm{BHU} / 15 / 511$
4: BHU/15/465
L7: BHU/15/513
L: BHU/15/51
L9:BHU/16/70
$\mathrm{L} 8: \mathrm{BHU} / 16 / 89$
$\mathrm{L} 11: \mathrm{BHU} / 16 / 9$

Fig. 4: 2\% Agarose Gel Electrophoresis for MYBPC3 having amplicon size 953 bp

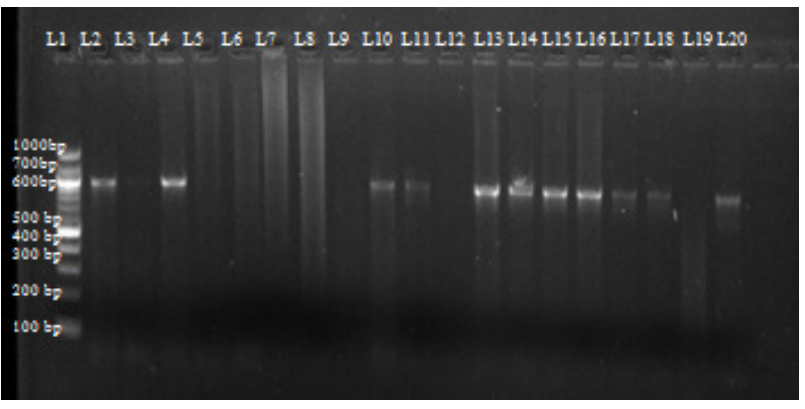
L1: Ladder
L2: BHU/15/408
L3: BHU/15/423
4: BHU/15/450
L5: BHU/15/453
L7: BHU/15/455
L6: BHU/15/454
L9: BHU/15/512
L8: $\mathrm{BHU} / 15 / 465$
L11: BHU/16/8
10: $\mathrm{BHU} / 15 / 608$
L13: BHU/16/39
L12: BHU/16/9
L15: BHU/16/59
L17: BHU/16/ 89
L14: BHU/16/58
L16: BHU/16/70
L18: BHU/16/104
L19: BHU/16/258
L20: BHU/16/345

Fig. 5: 2\% Agarose Gel Electrophoresis for MYBPC3 having amplicon size 953 bp
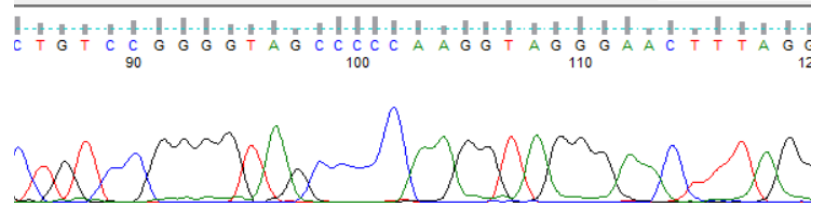

Fig. 6: Representative sequence electropherograms showing $c .36243625$ ins $C$ substitution in patient $B H U / 15 / 450$ in MYBPC3 gene

\begin{tabular}{|c|c|c|}
\hline Species/Abbrv & Group Name & $* * * * * * * * * * * * * * * * * * * * * * * * * * * * * * * * * * *$ \\
\hline 1. C1_MYBDC3F & & ctgctemectatccgegmagcccc-ahggtagg \\
\hline 2. NC_- $000011.10: C 47332753-47331801$ HHomo_sap: & & CTGCTGTGCTGICCGGGGTAGCCCC-AAGGTAGG \\
\hline 3. 2_MYBPC3F & & CTECTGTGCTGICCGGGGIAGCCCCCAAGGTAGG \\
\hline
\end{tabular}

Fig. 7: Multiple sequence alignment of MYBPC 3 gene in patient $\mathrm{BHU} / 15 / 450$ with control sample. 
Mutational analysis of MYBPC3 gene......

Table 1: Showing various synonymous and non-synonymous mutations

\begin{tabular}{|c|c|c|c|c|}
\hline S. No. & Genomic position & Patient ID & $\begin{array}{c}\text { Aminoacid } \\
\text { change }\end{array}$ & Mutation taster \\
\hline & \multicolumn{4}{|l|}{ MYBPC3 } \\
\hline 1 & Chr 11:47354160C>A & $\mathrm{BHU} / 15 / 407$ & G1195V & rs730880595 \\
\hline 2 & Chr 11: $47354127>47354128$ ins C & $\mathrm{BHU} / 15 / 408$ & S1207 & $\begin{array}{l}\text { HMGD CM057198, } \\
\text { CM068014 }\end{array}$ \\
\hline 3 & Chr11: $47354121 \mathrm{G}>\mathrm{C}$ & $\mathrm{BHU} / 15 / 408$ & P1208R & $\begin{array}{l}\text { SINGLE BASE } \\
\text { EXCHANGE,CDS }\end{array}$ \\
\hline 4 & Chr 11: 47354072_4735407 ins C & $\mathrm{BHU} / 15 / 408$ & & INTRON VARIANT \\
\hline 5 & Chr 11:47354038_47354039 ins G & $\mathrm{BHU} / 15 / 408$ & & INTRON VARIANT \\
\hline 6 & Chr 11: $47353899 \mathrm{C}>\mathrm{T}$ & $\mathrm{BHU} / 15 / 408$ & $\begin{array}{l}\text { SINGLE BASE } \\
\text { EXCHANGE }\end{array}$ & rs11039186 \\
\hline 7 & Chr 11: 47353536_47353537 insA & $\mathrm{BHU} / 15 / 408$ & & INTRON VARIANT \\
\hline 8 & Chr 11: 47353509_47353510 ins A & $\mathrm{BHU} / 15 / 408$ & & INTRON VARIANT \\
\hline 9 & Chr 11: 47353464_47353465 insA & $\mathrm{BHU} / 15 / 408$ & & INTRON VARIANT \\
\hline 10 & Chr 11: 47353715C>A & $\mathrm{BHU} / 15 / 423$ & R1241I & CDS \\
\hline 11 & Chr 11: 47353647A>C & $\mathrm{BHU} / 15 / 423$ & C1264G & CDS \\
\hline 12 & Chr 11: 47353626G>T & $\mathrm{BHU} / 15 / 423$ & CDS & HGMD CM086866 \\
\hline 13 & Chr 11: 47354102C>A & $\mathrm{BHU} / 15 / 424$ & & INTRON VARIANT \\
\hline 14 & Chr 11: $47354090 \mathrm{C}>\mathrm{T}$ & $\mathrm{BHU} / 15 / 424$ & & INTRON VARIANT \\
\hline 15 & Chr 11: 47354019_47354020 insA & $\mathrm{BHU} / 15 / 424$ & & INTRON VARIANT \\
\hline 16 & Chr 11: 47353977_47353978 insC & $\mathrm{BHU} / 15 / 424$ & & INTRON VARIANT \\
\hline 17 & Chr 11: 47353948A $>C$ & $\mathrm{BHU} / 15 / 424$ & & INTRON VARIANT \\
\hline 18 & Chr 11: 47353536T >A & $\mathrm{BHU} / 15 / 424$ & & INTRON VARIANT \\
\hline 19 & Chr 11: 47353502>A & $\mathrm{BHU} / 15 / 424$ & & INTRON VARIANT \\
\hline 20 & Chr 11: 47353496G>C & $\mathrm{BHU} / 15 / 424$ & & INTRON VARIANT \\
\hline 21 & Chr 11:47353464_47353465 insA & $\mathrm{BHU} / 15 / 424$ & & INTRON VARIANT \\
\hline 22 & Chr 11: 47353453T>C & $\mathrm{BHU} / 15 / 424$ & & INTRON VARIANT \\
\hline 23 & Chr 11: 47354119_47354120 insG & $\mathrm{BHU} / 15 / 425$ & K1209Q & $\begin{array}{l}\text { rs397516029 } \\
\text { FRAMESHIFT }\end{array}$ \\
\hline 24 & Chr 11: 47354095_47354096 insC & $\mathrm{BHU} / 15 / 425$ & & INTRON VARIANT \\
\hline 25 & Chr 11: 47354068G>A & $\mathrm{BHU} / 15 / 425$ & & $\begin{array}{c}\text { INTRON VARIANT } \\
\text { rs3729802 }\end{array}$ \\
\hline 26 & Chr 11: 47354040G>T & $\mathrm{BHU} / 15 / 425$ & & INTRON VARIANT \\
\hline 27 & Chr 11: 47353498G>A & $\mathrm{BHU} / 15 / 425$ & & $\begin{array}{l}\text { INTRON VARIANT } \\
\text { rs2290146 }\end{array}$ \\
\hline 28 & Chr 11: 47354119_47354120 insG & $\mathrm{BHU} / \mathbf{1 5 / 4 5 0}$ & K1209Q & rs397516029 \\
\hline
\end{tabular}




\begin{tabular}{|c|c|c|c|c|}
\hline S. No. & Genomic position & Patient ID & $\begin{array}{c}\text { Aminoacid } \\
\text { change }\end{array}$ & Mutation taster \\
\hline & & & & FRAMESHIFT \\
\hline 29 & Chr 11: 47354068G $>A$ & $\mathrm{BHU} / 15 / 450$ & & $\begin{array}{c}\text { INTRON VARIANT } \\
\text { rs3729802 }\end{array}$ \\
\hline 30 & Chr 11: 47353536_47353537insA & $\mathrm{BHU} / 15 / 450$ & & INTRON VARIANT \\
\hline 31 & Chr 11: 47353498G>A & $\mathrm{BHU} / 15 / 450$ & & $\begin{array}{c}\text { INTRON VARIANT } \\
\text { rs2290146 }\end{array}$ \\
\hline 32 & Chr 11: 47353464_47353465 insA & $\mathrm{BHU} / 15 / 450$ & & INTRON VARIANT \\
\hline 33 & Chr 11:47353434_47353435 insA & $\mathrm{BHU} / 15 / 450$ & & INTRON VARIANT \\
\hline 34 & Chr 11: 47353464_47353465 insA & $\mathrm{BHU} / 15 / 464$ & & INTRON VARIANT \\
\hline 35 & Chr 11: 47354090 delC & $\mathrm{BHU} / 15 / 465$ & & INTRON VARIANT \\
\hline 36 & Chr 11: 47353502C>A & $\mathrm{BHU} / 15 / 465$ & & INTRON VARIANT \\
\hline 37 & Chr 11: 47353464_47353465insA & $\mathrm{BHU} / 15 / 465$ & & INTRON VARIANT \\
\hline 38 & Chr 11: 47353453_4735345 insT & $\mathrm{BHU} / 15 / 465$ & & INTRON VARIANT \\
\hline 39 & Chr 11:47354090delC & $\mathrm{BHU} / 15 / 608$ & & INTRON VARIANT \\
\hline 40 & Chr 11:47354065C>T & $\mathrm{BHU} / 15 / 608$ & & INTRON VARIANT \\
\hline 41 & Chr 11:47353536_47353537insA & $\mathrm{BHU} / 15 / 608$ & & INTRON VARIANT \\
\hline 42 & Chr 11:47353485_47353486 insA & $\mathrm{BHU} / 15 / 608$ & & INTRON VARIANT \\
\hline 43 & Chr 11: 47353464_47353465insA & $\mathrm{BHU} / 15 / 608$ & & INTRON VARIANT \\
\hline 44 & Chr 11: $47353457-47353458$ ins A & $\mathrm{BHU} / 15 / 608$ & & INTRON VARIANT \\
\hline 45 & Chr 11: $47353745 \mathrm{C}>\mathrm{T}$ & $\mathrm{BHU} / 16 / 08$ & $\mathrm{~S} 1231 \mathrm{~N}$ & HGMD Cl014153 \\
\hline 46 & Chr 11:47353741C>T & $\mathrm{BHU} / 16 / 08$ & CDS & \\
\hline 47 & Chr 11:47353496G>C & $\mathrm{BHU} / 16 / 08$ & & INTRON VARIANT \\
\hline 48 & Chr 11:47354093delG & $\mathrm{BHU} / 16 / 39$ & & INTRON VARIANT \\
\hline 49 & Chr 11: $47354085 \mathrm{G}>\mathrm{A}$ & $\mathrm{BHU} / 16 / 39$ & & INTRON VARIANT \\
\hline 50 & Chr 11: $47353899 \mathrm{C}>\mathrm{T}$ & $\mathrm{BHU} / 16 / 39$ & & rs11039186 \\
\hline 51 & Chr 11: 47353434_47353435insA & $\mathrm{BHU} / 16 / 39$ & & INTRON VARIANT \\
\hline 52 & Chr 11: 47354038_47354039 insG & $\mathrm{BHU} / 16 / 58$ & & INTRON VARIANT \\
\hline 53 & Chr 11: 47353536_47353537 insA & $\mathrm{BHU} / 16 / 58$ & & INTRON VARIANT \\
\hline 54 & Chr 11: 47353498G $>A$ & $\mathrm{BHU} / 16 / 58$ & & rs2290146 \\
\hline 55 & Chr 11: 47353464_47353465 insA & $\mathrm{BHU} / 16 / 58$ & & INTRON VARIANT \\
\hline 56 & Chr 11: 47353874A>T & $\mathrm{BHU} / 16 / 58$ & & INTRON VARIANT \\
\hline 57 & Chr 11: 47353864G>C & $\mathrm{BHU} / 16 / 58$ & & INTRON VARIANT \\
\hline 58 & Chr 11: 47353756G $>A$ & $\mathrm{BHU} / 16 / 58$ & CDS & \\
\hline 59 & Chr 11: 47353459_47353460 insA & $\mathrm{BHU} / 16 / 58$ & & INTRON VARIANT \\
\hline 60 & Chr 11: 47353451_47353452 insC & $\mathrm{BHU} / 16 / 58$ & & INTRON VARIANT \\
\hline
\end{tabular}


Mutational analysis of MYBPC3 gene......

\begin{tabular}{|c|c|c|c|c|}
\hline S. No. & Genomic position & Patient ID & $\begin{array}{c}\text { Aminoacid } \\
\text { change }\end{array}$ & Mutation taster \\
\hline 61 & Chr 11:47354093delG & $\mathrm{BHU} / 16 / 59$ & & INTRON VARIANT \\
\hline 62 & Chr 11: $47354090 C>G$ & $\mathrm{BHU} / 16 / 59$ & & INTRON VARIANT \\
\hline 63 & Chr 11: 47353899C>T & $\mathrm{BHU} / 16 / 59$ & & rs11039186 \\
\hline 64 & Chr 11: 47353457_47353458 insA & $\mathrm{BHU} / 16 / 59$ & & INTRON VARIANT \\
\hline 65 & Chr 11: $47353899 \mathrm{C}>\mathrm{T}$ & $\mathrm{BHU} / 16 / 59$ & & rs11039186 \\
\hline 66 & Chr 11: 47354090deLC & $\mathrm{BHU} / 16 / 70$ & & INTRON VARIANT \\
\hline 67 & Chr 11: 47354068G>A & $\mathrm{BHU} / 16 / 70$ & & rs3729802 \\
\hline 68 & Chr 11: 47353498G>A & $\mathrm{BHU} / 16 / 70$ & & rs2290146 \\
\hline 69 & Chr 11:47353457_47353458 insA & $\mathrm{BHU} / 16 / 70$ & & INTRON VARIANT \\
\hline 70 & Chr 11: 47353434_47353435 insA & $\mathrm{BHU} / 16 / 70$ & & INTRON VARIANT \\
\hline 71 & Chr11:47353418_47353419 insT & $\mathrm{BHU} / 16 / 70$ & & INTRON VARIANT \\
\hline 72 & Chr11:47354119_47354120 insG & BHU/16/89 & K1209Q & $\begin{array}{c}\text { rs397516029 } \\
\text { FRAMESHIFT PRESENT }\end{array}$ \\
\hline 73 & Chr11:47354090_47354090 delC & $\mathrm{BHU} / 16 / 89$ & & INTRON VARIANT \\
\hline 74 & Chr11:47354068G>A & $\mathrm{BHU} / 16 / 89$ & & rs3729802 \\
\hline 75 & Chr11:47353498G>A & $\mathrm{BHU} / 16 / 89$ & & $\begin{array}{l}\text { INTRON VARIANT } \\
\text { rs2290146 }\end{array}$ \\
\hline 76 & Chr11:47353434_47353435insA & $\mathrm{BHU} / 16 / 89$ & & INTRON VARIANT \\
\hline
\end{tabular}

\section{DISCUSSION}

Dilated cardiomyopathy is most commonly associated with various other heart disorders. The most common associated disorder is congestive heart failure. In DCM there is ventricular chamber enlargement, the ventricular walls become thin and there is depressed left ventricular systolic function. It is most commonly idiopathic or familial. There may be various modes of inheritance i.e. autosomal dominant, autosomal recessive, X-linked or mitochondrial mode of inheritance.

Present study combined with previous studies, provides extensive data of association with the genetic factors causing DCM. We used Sanger based sequencing which is still the gold standard for sequencing sensitivity and specificity. In present study Exon 32-34 of MYBPC 3 gene was sequenced to identify possible genetic cause of dilated cardiomyopathy. Mutations in the gene for cardiac myosin-binding protein $\mathrm{C}$ account for main genetic cause of maximum cases of familial hypertrophic cardiomyopathy and dilated cardiomyopathy. In present study various synonymous and nonsynonymous variations had been observed.

In MYBPC3 gene, several intronic variations observed. Total 76 intronic variations were reported. Intronic variation does not alter protein structure but it may change splice site that may affect the protein structure and function. In three (BHU/15/425, $\mathrm{BHU} / 15 / 450, \mathrm{BHU} / 16 / 89$ ) patients disease causing variant c3624_3625insC (rs397516029, HMGD CD0910628) was reported in MYBPC3 gene. Same variation was reported by Hershberger et al., 2010 [8].

Some novel variant reported may lead to DCM. Insertion of $G$ at 47354119_47354120 position reported lead to a frameshift mutation in three subjects. Several missence variants were also observed $\quad 47354121 \mathrm{G}>\mathrm{C}, \quad 47353899 \mathrm{C}>\mathrm{T}$, $47353715 \mathrm{C}>\mathrm{A}, 47353647 \mathrm{~A}>\mathrm{C}, 47353626 \mathrm{G}>\mathrm{T}$ that are present in coding region and may lead to alteration in protein structure and function. Because of these variations are present in $\mathrm{C}$ terminal domain of protein that play very important role. 
Cardiac myosin-binding protein C (MYBPC3) is arrayed transversely in sarcomere A-bands and binds myosin heavy chain in thick filaments and in elastic filaments. Phosphorylation of this protein appears to modulate contraction [9]. The prognosis in patients with dilated cardiomyopathy is considered to be poor as morbidity and mortality rates are very high. Moreover, DCM is the chief indication for heart transplantation. So, it is important to diagnose the disease early for better treatment.

Evidence from previous study reported that MYBPC3 play important role in cardiac contraction and responsible for pathogenesis of dilated cardiomyopathy [10].

\section{CONCLUSION}

The identification of frequent genetic transmission of dilated cardiomyopathy provides an important tool for the study of pathogenesis of this disease, which is a frequent cause of admission to the hospital and of heart failure. Molecular genetic techniques help to identify the gene causing familial dilated cardiomyopathy and can be used to study the effects of altered gene product whether it is frameshit mutation or missense variation. Whereas early features of dilated cardiomyopathy can be identified by echocardiography or electrocardiogram or magnetic resonance imaging. The genetic as well as imaging information together provides accurate status of disease which helps in the management and prognosis of dilated cardiomyopathy. As soon as the genetic cause of dilated cardiomyopathy is diagnosed, the patient should undergo genetic counselling. It includes family history, education regarding transmission, benefits of regular cardiac screening tests. Multidisciplinary medical care for DCM includes genetic counsellors, cardiologists, medical geneticists.

In present study, mutational analysis of 32-34 exons of MYBPC3 gene and 14-15 exon of TNNT2 gene had been done. Various synonymous and nonsynonymous variations had been reported. Several intronic variation, frameshift mutation and missense variation are reported in both genes. That suggest these variation may be responsible for pathogenesis of dilated cardiomyopathy in patients of North Indian population. So, all families having familial transmission of dilated cardiomyopathy must have essential knowledge regarding the disease.

Funding Agency: The project was funded by Indian Council of Medical Research, New Delhi.

\section{REFERENCES}

1. Goodwin JF: The frontiers of cardiomyopathy. Br Heart J. 1982; 48:1-18.

2. Mestroni L, Rocco C, Gregori D, Sinagra G, Lenarda A, Miocic S, Vatta M, Pinamonti B, Muntoni F, Caforio AL, McKenna WJ, Giacca M, Camerini. Familial dilated cardiomyopathy: evidence for genetic and phenotypic heterogeneity. Journal of the American College of Cardiology. 1999; 34(1): 181-190.

3. Taylor MRG, Carniel E, Mestroni L. Cardiomyopathy, Familial Dilated. Orphanet Journal of Rare Diseases. 2006; I:27; 17501172-1-27.

4. Henry WL, Gardin JM, Ware JH. Echocardiographic measurements in normal subjects from infancy to old age. Circulation. 1980; 62: 1054-1061.

5. Report of the WHO/ISFC Task Force on the definition and classification of cardiomyopathies. Br Heart J. 1980; 44:672-4.

6. Van Driest SL, Vasile VC, Ommen SR, Will ML, Tajik AJ, Gersh BJ, Ackerman MJ. Myosin binding protein C mutations and compound heterozygosity in hypertrophic cardiomyopathy. J Am Coll Cardiol. 2004; 44:1903-10.

7. https://ghr.nlm.nih.gov/gene/MYBPC3\#location.

8. Ray E. Hershberger, Ana Morales, and Jill D. Siegfried. Clinical and genetic issues in dilated cardiomyopathy: A review for genetics professionals. Genet Med. 2010; 12(11): 655- 667.

9. Lisa Dellefave and Elizabeth M. McNally. The genetics of dilated cardiomyopathy. Current Opinion in Cardiology. 2010; 25:198-204.

10. Mestroni L, Rocco C, Gregori D et al. Familial dilated cardiomyopathy: evidence for genetic and phenotypic heterogeneity. Heart Muscle Disease Study Group. Journal of the American College of Cardiology. 1999; 34 (1): 181-190. 